The Role of Disk-Halo Interaction in Galaxy Evolution: Outflow vs Infall? Editor

EAS Publications Series, Vol. ?, 2018

\title{
IONIZATION OF INFALLING GAS
}

\author{
L. M. Haffner ${ }^{1}$, A.K. Duncan, S.M. Hoffman, G.J. Madsen ${ }^{2}$, A.S. Hill ${ }^{1}$ \\ and R.J. Reynolds ${ }^{1}$
}

\begin{abstract}
H} \alpha$ emission from neutral halo clouds probes the radiation and hydrodynamic conditions in the halo. Armed with such measurements, we can explore how radiation escapes from the Galactic plane and how infalling gas can survive a trip through the halo. The Wisconsin H-Alpha Mapper (WHAM) is one of the most sensitive instruments for detecting and mapping optical emission from the ISM. Here, we present recent results exploring the ionization of two infallling highvelocity complexes. First, we report on our progress mapping $\mathrm{H} \alpha$ emission covering the full extent of Complex A. Intensities are faint $\left(<100 \mathrm{mR} ; \mathrm{EM}<0.2 \mathrm{pc} \mathrm{cm}^{-6}\right)$ but correlate on the sky and in velocity with 21-cm emission. Second, we explore the ionized component of some Anti-Center Complex clouds studied by Peek et al. (2007) that show dynamic shaping from interaction with the Galactic halo.
\end{abstract}

\section{Complex A}

HVC Complex A is a distant ( $>8 \mathrm{kpc}$, Wakker et al. 2003; $<10 \mathrm{kpc}$, van Woerden et al. 1999) collection of infalling neutral gas $\left(v_{\mathrm{LSR}} \sim-200\right.$ to $\left.-130 \mathrm{~km} \mathrm{~s}^{-1}\right)$. Its metallicity and origin are still uncertain, although a detection of $\mathrm{O}$ I by Kunth et al. (1994) suggests neutral oxygen is $\leq 0.1$ solar (Wakker, 2001). Figure 1 shows the 21-cm emission from the complex. Tufte et al. (1998) provides the first measurement of $\mathrm{H} \alpha$ in the complex toward cores A III $(80 \mathrm{mR})$ and A IV $(90 \mathrm{mR})$.

In Figure 1 we present the first comprehensive map of $\mathrm{H} \alpha$ emission in the region. The map is composed of over 1600 WHAM one-degree beams, processed similar to the WHAM Northern Sky Survey (Haffner et al., 2003). The image is

WHAM and its research efforts are supported by National Science Foundation awards AST0204973 and AST-0607512. AKD received support through REU site award AST-0453442. GJM acknowledges support from U. Sydney through a University Postdoctoral Fellowship.

${ }^{1}$ Department of Astronomy, University of Wisconsin, Madison, WI, USA

2 School of Physics, The University of Sydney, NSW 2006, Australia 


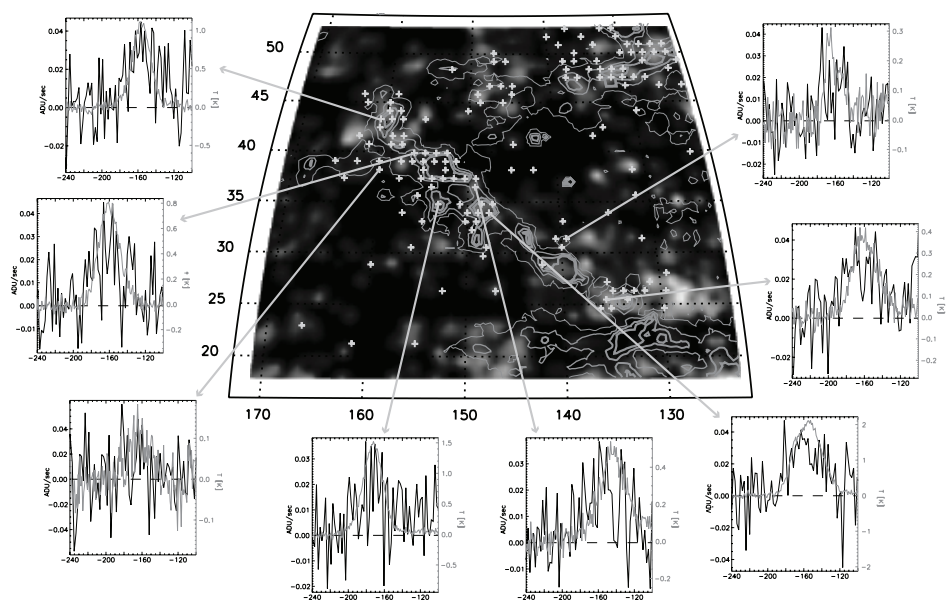

Fig. 1. Overlay of 21-cm contours $\left(1,3,5\right.$, and $7 \times 10^{19} \mathrm{~cm}^{-2}$; LDS, 0.5 beam) on an $\mathrm{H} \alpha$ intensity image (black, $<20 \mathrm{mR}$, white, $>90 \mathrm{mR}$; WHAM, $1^{\circ}$ beam) in the direction of Complex A. Axes are in Galactic degrees. White crosses show locations where means of fitted neutral and ionized emission components are close in velocity $\left(\Delta v<10 \mathrm{~km} \mathrm{~s}^{-1}\right)$. Spectra show example profiles from some of these directions ( $\mathrm{H} \alpha$, black; 21-cm, gray).

then constructed by integrating over velocities that sample Complex A ( -220 to $-110 \mathrm{~km} \mathrm{~s}^{-1}$ ). First presented by Duncan et al. (2005), we have since doubled the total integration (to $120 \mathrm{~s}$ ) for about half of the sightlines, mostly distributed along the Complex A chain. Due to considerable atmospheric line density at intensities $\sim 10-100 \mathrm{mR}$, baseline fitting is the major source of uncertainty. Combining cleaned spectra obtained on multiple nights lessens the impact of any systematic contaminants. Nonetheless, the resulting map still shows very low contrast compared to the 21-cm map of the region, here from the Leiden-Dwingeloo survey (LDS, Hartmann and Burton, 1997). Most detected components have $\mathrm{H} \alpha$ intensities of $20-80 \mathrm{mR}\left(\mathrm{EM} \sim 0.04-0.18 \mathrm{pc} \mathrm{cm}^{-6}\right.$, if $\left.T_{e}=8000 \mathrm{~K}\right)$.

To study the relationship between the ionized and neutral components, we developed a simple, automated, Gaussian fitting algorithm to characterize positive detections in $\mathrm{H} \alpha$ and 21-cm spectra. Focusing on lines of sight that show a strong kinematic relationship between the two gas components, we identify directions in Figure 1 (white crosses) where the fitted mean velocity of the $\mathrm{H} \alpha$ and 21-cm component differ by less than $10 \mathrm{~km} \mathrm{~s}^{-1}$. Plots comparing the two emission spectra toward a representative sample of these directions are also shown. Exploring the data in the spectral domain adds considerable confidence that the WHAM data is tracing high-velocity gas, especially toward directions with measurable $\mathrm{H}$ I column density. The correspondence between the neutral and ionized gas velocities seen in the selected spectra here continue in the larger statistical sample. The two phases are kinematically linked, as seen in other IVC and HVC studies (see Haffner et al. 

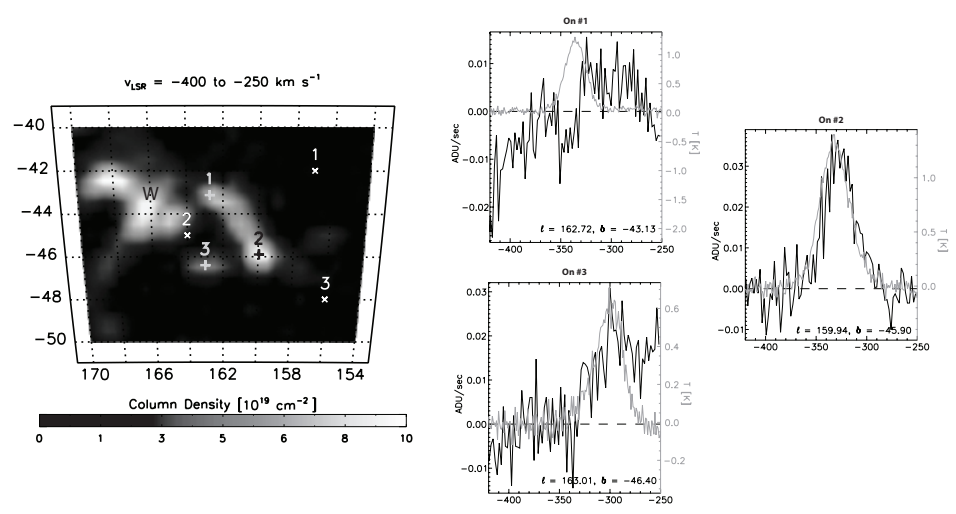

Fig. 2. (left) 21-cm emission from LDS in the direction of a few high-latitude AntiCenter HVCs. The location of the WHAM observations $(+$, on; $\times$, off $)$ are shown. The black "W" denotes the approximate location of the $\mathrm{H} \alpha$ detection (40 mR) reported by Weiner et al. (2001). (right) $\mathrm{H} \alpha$ spectra (black) with background subtracted towards the three "on" directions. The corresponding average 21-cm spectrum (gray) from LDS within the WHAM one-degree beam is also shown.

2001 and Haffner 2005). H $\alpha$ intensity, on the other hand, does not correlate (or anti-correlate) with $\mathrm{H}$ I column density. As with other studied complexes, the column density exhibits much larger variations over the extent of the HVC than the $\mathrm{H} \alpha$ intensity. Here, the neutral column spans about a factor of 10 to 20 while the emission measure varies by a factor of 3 to 5 .

\section{Anti-Center Clouds}

Peek et al. (2007) present 21-cm observations from Arecibo $\left(\sim 3^{\prime}\right)$ of a very highvelocity $\left(v_{\mathrm{LSR}} \sim-330 \mathrm{~km} \mathrm{~s}^{-1}\right)$ segment of the Anti-Center complex. Based on the morphology of the main feature and several red-shifted $\left(\Delta v \sim 30 \mathrm{~km} \mathrm{~s}^{-1}\right)$, smaller clouds, they suggest that the gas is being dynamically shaped and stripped away by interaction with the halo. Weiner et al. (2001) report $\mathrm{H} \alpha$ emission of about $40 \mathrm{mR}$ toward another cloud in the subcomplex (HVC 165-43-280; see Figure 2). Based on this emission and a rough model of ionizing flux in the halo, they locate the HVC between about 10 and $20 \mathrm{kpc}$ away. Peek et al. (2007) find that their drag model predicts a consistent distance.

Three "on" and three "off" directions (labeled in Figure 2) were identified as the initial WHAM targets. Each location was observed for $120 \mathrm{~s}$ per exposure over two nights separated by a month, with "on" and "off" targets interleaved to aid atmospheric line removal. The total exposure time per direction is $1680 \mathrm{~s}$. None of the "off" directions appear to contain Galactic emission, so a single, very high signal-to-noise "off" is created for each night and subtracted from each "on" 
4 The Role of Disk-Halo Interaction in Galaxy Evolution: Outflow vs Infall?

direction. Results are shown in Figure 2 with the WHAM spectrum in black and the $\mathrm{H}$ I spectrum from LDS shown in gray.

$\mathrm{H} \alpha$ emission is clearly detected in Directions $2(60 \mathrm{mR})$ and $3(35-90 \mathrm{mR})$ with velocity profiles similar to those of the 21-cm emission. Direction 1 shows no clear emission at the location of the $\mathrm{H}$ I velocity. Although the baseline is far from flat, the range of intensities limits any possible emission to no more than one-third that of Direction $2(20 \mathrm{mR})$. Direction 3 hints at red-shifted emission or a second component. This asymmetry is present in both nights when examined separately. Integrating over the whole velocity window gives a lower limit of 90 $\mathrm{mR}$, while assuming a $30 \mathrm{~km} \mathrm{~s}^{-1} \mathrm{FWHM}$ as measured in Direction 2 gives $35 \mathrm{mR}$ for a component centered near $v_{\mathrm{LSR}}=-290 \mathrm{~km} \mathrm{~s}^{-1}$.

\section{References}

Duncan, A. K., Haffner, L. M., Madsen, G. J., and Reynolds, R. J.: 2005, in Bulletin of the American Astronomical Society, Vol. 37, p. 1256

Haffner, L. M.: 2005, in R. Braun (ed.), Extra-Planar Gas, Vol. 331 of Astronomical Society of the Pacific Conference Series, p. 25

Haffner, L. M., Reynolds, R. J., and Tufte, S. L.: 2001, ApJ 556, L33

Haffner, L. M., Reynolds, R. J., Tufte, S. L., Madsen, G. J., Jaehnig, K. P., and Percival, J. W.: 2003, ApJS 149, 405

Hartmann, D. and Burton, W. B.: 1997, Atlas of Galactic Neutral Hydrogen, Cambridge University Press, Cambridge; New York

Kunth, D., Lequeux, J., Sargent, W. L. W., and Viallefond, F.: 1994, A\&A A 282, 709

Peek, J. E. G., Putman, M. E., McKee, C. F., Heiles, C., and Stanimirović, S.: 2007, ApJ 656, 907

Tufte, S. L., Reynolds, R. J., and Haffner, L. M.: 1998, ApJ 504, 773

van Woerden, H., Schwarz, U. J., Peletier, R. F., Wakker, B. P., and Kalberla, P. M. W.: 1999, Nat 400, 138

Wakker, B. P.: 2001, ApJS 136, 463

Wakker, B. P., Savage, B. D., Sembach, K. R., Richter, P., Meade, M., Jenkins, E. B., Shull, J. M., Ake, T. B., Blair, W. P., Dixon, W. V., Friedman, S. D., Green, J. C., Green, R. F., Kruk, J. W., Moos, H. W., Murphy, E. M., Oegerle, W. R., Sahnow, D. J., Sonneborn, G., Wilkinson, E., and York, D. G.: 2003, ApJS 146, 1

Weiner, B. J., Vogel, S. N., and Williams, T. B.: 2001, in J. E. Hibbard, M. Rupen, and J. H. van Gorkom (eds.), Gas and Galaxy Evolution, Vol. 240 of Astronomical Society of the Pacific Conference Series, p. 515 